\title{
BMJ Open The NeuroAiD Safe Treatment (NeST) Registry: a protocol
}

\author{
Narayanaswamy Venketasubramanian, ${ }^{1}$ Ramesh Kumar, ${ }^{2}$ Lyna Soertidewi, ${ }^{3}$ \\ Azizi Abu Bakar, ${ }^{2}$ Carine Laik, ${ }^{4}$ Robert Gan ${ }^{4}$
}

\section{To cite:}

Venketasubramanian $\mathrm{N}$, Kumar R, Soertidewi L, et al. The NeuroAiD Safe Treatment (NeST) Registry: a protocol. BMJ Open 2015;5:e009866. doi:10.1136/bmjopen-2015009866

- Prepublication history for this paper is available online. To view these files please visit the journal online (http://dx.doi.org/10.1136/ bmjopen-2015-009866).

Received 31 August 2015 Revised 19 October 2015 Accepted 26 October 2015

\section{(a) CrossMark}

${ }^{1}$ Department of Neurology, Raffles Neuroscience Centre, Raffles Hospital, Singapore, Singapore

${ }^{2}$ Department of

Neurosurgery, Faculty of Medicine, University Kebangsaan Malaysia Medical Centre, Kuala Lumpur, Malaysia

${ }^{3}$ Department of Neurology, National Brain Center Hospital, Jakarta, Indonesia ${ }^{4}$ Moleac, Singapore,

Singapore

Correspondence to Dr Robert Gan; robert.gan@moleac.com

\section{ABSTRACT}

Introduction: NeuroAiD (MLC601, MLC901), a combination of natural products, has been shown to be safe and to aid neurological recovery after brain injuries. The NeuroAiD Safe Treatment (NeST) Registry aims to assess its use and safety in the real-world setting.

Methods and analysis: The NeST Registry is designed as a product registry that would provide information on the use and safety of NeuroAiD in clinical practice. An online NeST Registry was set up to allow easy entry and retrieval of essential information including demographics, medical conditions, clinical assessments of neurological, functional and cognitive state, compliance, concomitant medications, and side effects, if any, among patients on NeuroAiD. Patients who are taking or have been prescribed NeuroAiD may be included. Participation is voluntary. Data collected are similar to information obtained during standard care and are prospectively entered by the participating physicians at baseline (before initialisation of NeuroAiD) and during subsequent visits. The primary outcome assessed is safety (ie, non-serious and serious adverse event), while compliance and neurological status over time are secondary outcomes. The in-person follow-up assessments are timed with clinical appointments. Anonymised data will be extracted and collectively analysed. Initial target sample size for the registry is 2000 . Analysis will be performed after every 500 participants entered with completed follow-up information.

Ethics and dissemination: Doctors who prescribe NeuroAiD will be introduced to the registry by local partners. The central coordinator of the registry will discuss the protocol and requirements for implementation with doctors who show interest. Currently, the registry has been approved by the Ethics Committees of Universiti Kebangsaan Malaysia (Malaysia) and National Brain Center (Indonesia). In addition, for other countries, Ethics Committee approval will be obtained in accordance with local requirements.

Trial registration number: NCT02536079.

\section{INTRODUCTION}

MLC601 (NeuroAiD, Nu-rAiD) is a combination of 14 natural ingredients indicated as

\section{Strengths and limitations of this study}

Proactive collection of safety data in the realworld setting.

- Does not interfere with clinical decision-making of physicians and patients.

- Information and data elements collected closely mimic standard care.

- Uses a secured online data entry system.

- Biases may be difficult to control, no concurrent control arm for comparison.

treatment for post-stroke recovery widely used in China and in many countries in Asia. ${ }^{1}$ In Europe, a simplified formulation of the product, MLC901 (NeuroAid II, NurAiD II), consisting of nine herbal components, is available and will soon be available in Asian countries as well. ${ }^{1-5}$ Both formulations shall collectively be referred to as 'NeuroAiD' in this registry.

NeuroAiD efficacy and safety are supported by preclinical and clinical studies. Toxicity studies have confirmed the absence of significant toxic effects in animals. [1,2, on file in Moleac] The neuroprotective and neuroproliferative properties of NeuroAiD have been extensively elucidated in in vitro and in vivo experiments using animal and cellular models of focal and global ischaemia. ${ }^{1-4}$ What is remarkable are the effects of NeuroAiD on neurogenesis and neurorestoration beyond mere neuroprotection. In addition, its positive effects in traumatic brain injury (TBI) have recently been published, ${ }^{5}{ }^{6}$ and early preclinical data on its effect on amyloid precursor protein processing are emerging. ${ }^{7}$

The clinical data on NeuroAiD are most well reported in stroke. A systematic review of randomised clinical trials on NeuroAiD showed its benefits in improving functional outcomes and neurological deficits with 3 months treatment among patients with ischaemic stroke in the preceding 1 week to 6 months. $^{8-13}$ Subsequently, NeuroAiD was 
investigated in acute ischaemic stroke within $72 \mathrm{~h}$ of onset, which demonstrated the treatment effect to be larger in postacute and relatively more severe stroke at 3 months. ${ }^{14-17}$ The updated meta-analysis showed a pooled OR still in favour of NeuroAiD. ${ }^{15}$ Furthermore, treatment with NeuroAiD was associated with a reduction in risk of early vascular events after a stroke. ${ }^{18}$ Recently, the extension of this randomised, placebocontrolled, double-blind trial has provided evidence of its benefits on the long-term functional outcome persisting overtime up to 18 months with an excellent safety profile. $^{19} 20$

Case reports of the use of NeuroAiD in neurosurgical conditions have been the subject of presentations in international neurosurgical congresses. ${ }^{21}{ }^{22}$ Encouraging clinical data have been reported on its safety and potential efficacy as well in Alzheimer's disease. ${ }^{23}{ }^{24}$ In addition, there are several ongoing studies on the use of NeuroAiD in post-stroke cognitive impairment and TBI. ${ }^{25-27}$

Since 2001 when it was marketed in China, there have been minimal serious side effects reported to date with the use of NeuroAiD. The common side effects reported from NeuroAiD were mostly mild and transient. Excellent clinical safety has been demonstrated in published clinical trials which reported the more common adverse events (AEs) being gastrointestinal symptoms (nausea, vomiting, discomfort, diarrhoea, dry mouth), and headache. ${ }^{8-13}$ Safety studies in humans have shown that NeuroAiD, given alone or combined with aspirin, had no effect on clotting and coagulation. ${ }^{28}$ Furthermore, there was no effect on haematological, haemostatic and biochemical parameters, and/or ECG in normal patients and patients with stroke, even when started within $48 \mathrm{~h}$ of stroke onset. ${ }^{28-30}$

While the safety of NeuroAiD is well established in clinical studies, this registry will assess the safe use of NeuroAiD in the real-world setting.

\section{OBJECTIVE}

The primary objective of the registry is to evaluate the use and safety of NeuroAiD in the real-world setting. Participation is entirely voluntary. It is hoped that by patients' participation in the registry, more uniform and reliable information can be collected from participants who are actually taking open-label NeuroAiD.

\section{METHODS}

\section{Study design and subject eligibility}

The NeST Registry is a product-specific and safety outcome registry. Patient participation is entirely voluntary and an agreement will be obtained before participation (figure 1). The decision on the use of NeuroAiD is made following a discussion between the participant and the physician and only then will the option of participation in the registry be considered.
Patients may be included in the registry if they meet all of the inclusion and none of the exclusion criteria:

\section{Inclusion criteria}

- Male or female

- Any age

- Any patient who is taking or has been prescribed NeuroAiD for any duration as judged by the physician and/or the participant (Note: NeuroAiD is mainly indicated for patients with brain injuries. It is expected that most participants included in the registry would have a stroke, TBI or a hypoxic event to the brain. However, patients taking NeuroAiD for other medical or neurological conditions may likewise be included in the registry.)

- Agrees to be included in the registry and allows retrieval and analysis of data in accordance with local requirements.

\section{Exclusion criteria}

- Unwillingness to participate

- Contraindication to NeuroAiD



Figure 1 NeST Registry flow chart (GCS, Glasgow Coma Scale; mRS, modified Rankin Scale; NeST, NeuroAiD Safe Treatment; NIHSS, National Institutes of Health Stroke Scale; SOMCT, Short Orientation Memory Concentration Test). 


\section{Treatment}

Each $400 \mathrm{mg}$ capsule of MLC601 contains nine herbal ingredients (extracts of Radix astragali, Radix salvia miltiorrhizae, Radix paeoniae rubra, Rhizoma chuanxiong, Radix angelicae sinensis, Carthamus tinctorius, Prunus persica, Radix polygalae and Rhizoma acori tatarinowii) and five non-herbal components (Hirudo, Eupolyphaga seu steleophaga, Calculus bovis artifactus, Buthus martensii and Cornu saigae tataricae). MLC901 contains only herbal extracts. The product is available in capsule form and administered orally or the contents may be diluted in water and administrated via a gastric tube. The usual dosage is 4 capsules three times a day for MLC601 and 2 capsules three times a day for MLC901. The recommended treatment duration is 3 months. The capsules should be kept sealed and stored below $30^{\circ} \mathrm{C}$ in a dry place until opened for administration.

NeuroAiD is manufactured according to applicable control measures that ensure the consistency and quality of the product from batch to batch and adhere to good manufacturing practice. The active ingredients and finished product are subjected to full quality control testing for safety.

All participants are allowed to receive standard care and other therapies and treatments, including (but not limited to) blood pressure control, rehabilitation and other types of care deemed appropriate and as prescribed by their physician. There is no restriction to the use of any other treatment as recommended by the treating physician, although they should be recorded in the database.

\section{Data collection}

Data will be collected through an online data entry system (http://www.neuroaid.com/en/nest/). Contributors to the registry will be provided secured access accounts with username and password. Paper report forms are available if online submission is not possible, for example, internet downtime, computer malfunction, power outage, but online entry is the preferred mode of data collection. If paper forms are used, data must be written in a neat and legible manner, using a black or blue ballpoint pen to ensure the clarity of the reproduced copy of all completed forms which are signed and dated. The completed online or paper forms shall serve as the source document. No other medical record or source document will be required for this study.

The advantages of the online system are its accessibility from any clinic with an internet-connected computer and that non-conforming and missing required data are immediately corrected. While it requires an internetconnected computer, log-in access to the website and online data entry which may not be consistent with the clinician's practice, it is expected to save time in the long run by reducing the need for clarifying illegible handwriting, inconsistency of information, missing or non-conformant data, and other concerns inherent in using paper forms.
The registry is designed to minimise intrusion into usual clinical practice. However, there may be some disadvantages to a registry that is designed to be closely aligned with clinical practice. The limited data collected may not provide an opportunity for extensive analyses and follow-up visits may be hampered by the usual unpredictability and loss as seen in the real world. Factors such as local system of care, economics, practicalities and culture are expected to have a bigger impact on registries compared to clinical trials.

\section{Data elements}

Participants will undergo assessments at baseline (before start of NeuroAiD intake) and at months 1 ( \pm 7 days), 2 ( \pm 7 days) and 3 ( \pm 14 days) (table 1 ). Additional assessments may be performed if the participant is still on NeuroAiD. Information to be collected is meant to specifically address the objectives of the registry.

Data collected at baseline, immediately prior to or at the start of NeuroAiD treatment, will include:

General information

- Demographics information: date of birth, gender, ethnicity;

- The main diagnosis for taking NeuroAiD and the date of its onset;

- Other relevant medical conditions;

- The brand of NeuroAiD, date started and dose.

Baseline information

- National Institutes of Health Stroke Scale (NIHSS) $;^{31}$

- Glasgow Coma Scale (GCS) $;^{32}$

- Modified Rankin Scale (mRS) $;^{33}$

- Short Orientation Memory Concentration Test (SOMCT) $;^{34}$

- Concomitant medication: generic name and dose.

Data collected from months 1 to 3 (and any additional visits beyond month 3) will include compliance with intake of NeuroAiD and occurrence of any AE in addition to the other clinical assessments performed at baseline.

\section{Safety considerations}

\section{Definition of $\mathrm{AE}$ and serious $\mathrm{AE}$}

An $\mathrm{AE}$ is defined as any untoward medical occurrence in a person administered a product and which does not necessarily have a causal relationship with this treatment. An AE is considered serious (SAE) if it results in death, a persistent or significant disability, abortion, congenital anomaly, or birth defect, is life-threatening, or requires inpatient hospitalisation or prolongation of existing hospitalisation.

\section{Side effect (adverse drug reaction)}

A side effect is an effect, whether therapeutic or adverse, that is secondary to the one intended. It can also apply to beneficial, but unintended, consequences of the use of a treatment. For the purpose of this registry, a side effect (or adverse drug reaction) is any 
Table 1 Overview of information collected at baseline and subsequent visits

\begin{tabular}{|c|c|c|c|c|c|}
\hline Information collected & Baseline & $\begin{array}{l}\text { Follow-up } \\
\text { visit } 1\end{array}$ & $\begin{array}{l}\text { Follow-up } \\
\text { visit } 2\end{array}$ & $\begin{array}{l}\text { Follow-up } \\
\text { visit } 3\end{array}$ & $\begin{array}{l}\text { Additional } \\
\text { visits }\end{array}$ \\
\hline Demographics & $\mathrm{X}$ & & & & \\
\hline $\begin{array}{l}\text { Diagnosis and other medical } \\
\text { conditions }\end{array}$ & $x$ & & & & \\
\hline $\begin{array}{l}\text { NeuroAiD information and } \\
\text { compliance }\end{array}$ & $x$ & $X$ & $x$ & $x$ & $x$ \\
\hline NIHSS & $x$ & $x$ & $x$ & $x$ & $x$ \\
\hline GCS & $x$ & $x$ & $X$ & $x$ & $x$ \\
\hline $\mathrm{mRS}$ & $x$ & $x$ & $x$ & $x$ & $X$ \\
\hline SOMCT & $X$ & $\mathrm{X}$ & $X$ & $x$ & $X$ \\
\hline Concomitant medications & $x$ & $x$ & $x$ & $x$ & $x$ \\
\hline Side effects & & $\mathrm{X}$ & $x$ & $x$ & $X$ \\
\hline
\end{tabular}

unintended $\mathrm{AE}$ that is related to the use of the treatment, NeuroAiD. On the basis of causality as defined by the WHO-Uppsala Monitoring System, ${ }^{35}$ any AE that is considered by the treating physician as being possibly, probably or definitely related to NeuroAiD would be considered as a side effect.

\section{Reporting of $A E$ and side effects}

All AE or laboratory abnormalities that develop during the course of the patient's treatment will have to be reported in the study. AE should be reported as a diagnosis or syndrome or, if this is not possible, the specific symptom or abnormality may be entered. The start date of the onset of any sign or symptom of the event and stop date (date the side effect is considered to have resolved, if resolved) should be entered. Severity (as mild, moderate or severe) is recorded according to how the doctor, participant and/or caregiver deems the severity.

Side effects that are reported in the registry, especially those categorised as 'severe', will trigger a request for more medical information to ascertain details of the event and the need for reporting the event as serious adverse reaction to local regulatory authorities, if required.

\section{Follow-up of $\mathrm{AE}$}

Any AE must be followed until resolution, until the condition stabilises, until the event is explained, or until the participant is lost to follow-up. The physician is responsible for ensuring that standard medical diagnostic or therapeutic management, if any, is performed.

\section{Common side effects reported from NeuroAiD}

The common side effects reported from NeuroAiD use were mostly mild and transient. These include dry mouth, nausea, vomiting, abdominal discomfort, diarrhoea and headache. In many cases, the side effect resolves with reduction of the dose by half for 1 week and is resumed at the usual dose after resolution of the symptom.

\section{Ethical considerations}

The potential risks involved in participating in the registry are low as this is a non-interventional programme and not a clinical trial. The discussion and decision as regards the appropriate use of NeuroAiD in the participant would have been made prior to inclusion in the registry. The possible common side effects of the treatment and, in very rare cases, severe allergic reaction or unexpected life-threatening events will be explained. There is no restriction on other therapies recommended by the treating physician.

Currently, the registry has been approved by the ethics committees of the University Kebangsaan Malaysia and the National Brain Center (Indonesia). For other clinics, hospitals and countries participating in the future, appropriate approvals will be obtained in accordance with local health authority requirements. This should be ascertained and complied with for each site and country prior to conducting the registry. If institutional or country ethics approval is necessary for the registry, a written informed consent form approved by the appropriate committee must be obtained from the participant or the legal representative and documented in the database or medical records. All participants will need to provide at least a verbal consent for their anonymised data to be collected if written consent is waived by the approving authority.

If ethics committee approval was necessary for the initial implementation of the registry, this must also be obtained for any amendments made after initial ethics committee approval, except where necessary to eliminate an immediate hazard to participants, or when the change involves only logistical or administrative aspects of the registry (eg, change of personnel, change of telephone number).

Moleac acts as a central coordinator for the registry. No subject will be identified or included through Moleac. The protection of personal information of participants in the registry is described in the section below. 


\section{Data management}

The registry is a proactive industry-academic collaboration supported by Moleac, the manufacturer of NeuroAiD, and thereby ensures that appropriate safety information will reach stakeholders in a timely manner through its link with the pharmacovigilance system of Moleac. The online web-based data entry system is compliant with the Health Insurance Portability and Accountability Act. Data will be anonymised and maintained in a secured database in Moleac which is backed up regularly and accessible only to relevant personnel. Patients' identities will be recorded only as initials with identification numbers. Specific individual information in the registry will not be shared with other persons, entities or companies unless obligated by legal authorities. Collective information will be summarised and reviewed. These summaries may be presented to stakeholders (eg, physicians, regulatory authorities, attendees in a conference, etc) and/or published in scientific journals.

\section{Sample size}

The Registry must have enough sample size to be representative of the real-world setting and, at the same time, allow subgroups to be represented and meaningfully analysed. As an initial plan, the minimum target number of participants to be included in this registry is 2000 .

The 'rule of three', described by Hanley and Lippman-Hand ${ }^{36}$ in 1983, estimates the worst case that is compatible with the observed data and offers a quick interpretation of a specific event not being observed in a population. ${ }^{37}$ The rule estimates that in a large enough study (ie, $>30$ participants), if no event occurs, there is a $95 \%$ confidence that the event would not actually occur more often than once in $n / 3$ people, where $n$ is the sample size. Although this rule must be carefully applied, since it assumes that reporting of all events occurring in the study population is complete and that the study population is an accurate representation of the intended population, it provides some guidance regarding the registry size and interpretation of results.

\section{Statistical analysis and signal detection}

Descriptive statistics will be used to summarise data and will be reported as numbers, proportions, medians with corresponding ranges or means with corresponding SDs. Outcome assessments will be compared to baseline and previous observations.

Cases of side effects reported in the registry will be tabulated and individual case reports of side effects will be reviewed. Events and numbers will be compared with previously reported clinical trials and classified as 'expected' or unexpected'. Qualitative and, whenever applicable, quantitative signal detection will be performed. Signal detection may be accomplished by the Proportional Reporting Ratio and/or the Bayesian Confidence Propagation Neural Network. ${ }^{38-41}$ Both methods are based on a quantitative measure of disproportionality which compares the observed with the expected reports of a certain outcome-exposure combination. Disproportionality analyses may also be performed between MLC601 and MLC901 and/or different subgroups (eg, age, gender, underlying disease and severity). Since signal detection does not conclude causality and is only hypothesis generating, an event that appears to be product related may lead to further evaluation to examine whether the occurrence appears to be related to a specific treatment, a combination or sequence of treatments, or a particular dosage and/or duration of use. Other analyses deemed appropriate by the scientific committee and statistical consultant will also be performed.

\section{Periodic review}

Periodic summary reporting to the scientific committee and analyses will be performed on the database every 6 months or after every new 500 participants have been entered with complete follow-up information, whichever comes first.

\section{Registry administration and oversight}

Both hospital-based and community-based medical practitioners will be invited to collaborate and contribute to the registry (figure 2). National coordinators, who may be members of the scientific advisory group, will advise on national perspectives based on discussions with collaborators in the country. The central scientific advisory group, headed by a Chair, shall provide scientific advice to Moleac on data evaluation, safety assessment, reports and publications. Central coordination, including administrative support, maintenance of the database and online data collection tool, data management and

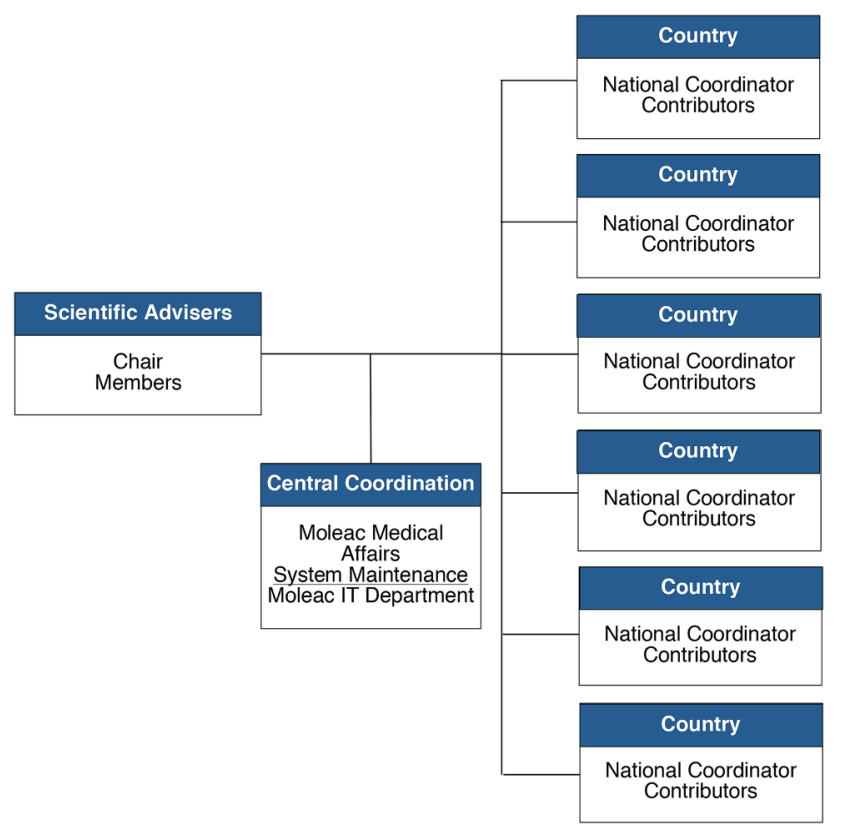

Figure 2 Organisational structure of oversight of NeuroAiD Safe Treatment (NeST) Registry. 
preparation of periodic reports, shall be provided by the medical affairs and information technology departments of Moleac.

\section{Closure of registry}

The Registry shall be closed when the planned number of participants has been reached and the scientific advisers agree that further inclusion is no longer relevant or necessary. The closure will occur in steps, starting with informing national coordinators and contributors of the plan to discontinue inclusion of any more participants and until after the follow-up of the last participant has been completed. The database will subsequently be locked on the basis of an agreed time frame with the scientific advisers.

\section{DISCUSSION}

The NeuroAiD Safe Treatment (NeST) Registry is designed as a product registry that would provide information on the use and safety of NeuroAiD in clinical practice. While the two formulations are not completely similar, the major ingredients are the same with similar pharmacological effects and indications for use. Thus, both formulations are included in this registry. Nonetheless, the formulation used will be identified in the database and will allow them to be analysed separately.

Doctors who prescribe NeuroAiD will be introduced to the registry by local partners. The medical affairs department of Moleac, acting as the central coordinator of the registry, will discuss the full protocol and requirements for implementation with the interested doctors. Contributing doctors will be medical practitioners who have no direct affiliation with the drug manufacturer, except through the use of the product, participation in clinical studies on NeuroAiD, and/or conduct of this registry.

Although spontaneous reporting is a cost-effective method of detecting product safety issues early, it is very much dependent on the initiative and motivation of the reporters and often leads to under-reporting. ${ }^{42}$ The absence of a system, protocols, tools and mandate can be barriers to such adverse drug reaction reporting. ${ }^{43}$ A registry can provide valuable information taking into account the different scenarios of product use in the real world (eg, switching therapies during follow-up, use of multiple products in combination or in sequence, different dosing, compliance, etc) that may not be possible in clinical trials. ${ }^{44}$ In contrast to a clinical trial where an active intervention intended to change a human subject's outcome is implemented and takes decisionmaking away from the physician and patient, medical decisions are strictly regulated by the study protocol and extensive data are collected for analyses, this registry uses an observational design and does not specify or assign treatments or therapies. On the other hand, biases in registries are difficult to control. Often, there is no concurrent control arm for comparison, participants are less homogeneous in characteristics, some data may be unavailable, and concomitant therapies can be widely variable.

Clinicians in the real-world setting make treatment recommendations based on medical judgement and existing evidence. They are often more preoccupied with more patient-related concerns with little time to spare on formalities of data collection. The NeST Registry, therefore, is designed to be as less intrusive to patient care as possible. There are few inclusion and exclusion criteria in an effort to include a broad range of participants to make the results more generalisable. Participants are observed as they present for care and the data collected reflect whatever tests, measurements and treatments a healthcare provider customarily uses. The data elements selected for the NeST Registry is a balance between ease of use for the respondents (ie, the busy clinicians), likelihood of complete data collection, relevance and reliability of the data domains. Established clinical standards, common definitions and validity of scales are considered in the selection of data elements. ${ }^{31-34}$

\section{SUMIMARY}

The NeST Registry is a proactive industry-academic collaboration to assess the use and safety of NeuroAiD in the real-world setting. An online entry system was set up to allow easy data entry and retrieval of clinical information. The registry can be a complementary route for more accurate pharmacovigilance, which is an important component of patient care and surveillance.

Acknowledgements Naw Freda and Saiful Najaib (Moleac) programmed the online system and maintain the integrity of the NeST Registry system.

Contributors NV, RK, LS, AAB, CL and RG have contributed substantially to the conceptualisation, design and writing of the study protocol. NV is chair while RK and LS are members of the NeST scientific advisory committee. RK and $A A B$ are the national coordinator and contributor, respectively, for NeST Malaysia. LS is the national coordinator for NeST Indonesia. CL and RG drafted the manuscript which was reviewed, corrected and approved by NV, RK, LS and $A A B$.

Funding This work is supported by Moleac Singapore Pte Ltd. The study design was jointly conceptualised by the scientific advisers of the registry and Moleac. Central coordination is provided by the medical affairs department of Moleac. Collaborating physicians collect and enter the anonymised subject data. Analysis and interpretation of the data, writing of the report and the decision to submit papers for publication is at the discretion of the scientific advisory committee, although Moleac will be informed of the plan and results

Competing interests NV and RK have received support for clinical trials on NeuroAiD and presentations at scientific meetings from Moleac. CL and RG are employees of Moleac.

Ethics approval Ethics committees of the University Kebangsaan Malaysia and the National Brain Center (Indonesia).

Provenance and peer review Not commissioned; externally peer reviewed.

Data sharing statement Any other additional details of the full protocol can be obtained from the corresponding author.

Open Access This is an Open Access article distributed in accordance with the Creative Commons Attribution Non Commercial (CC BY-NC 4.0) license, which permits others to distribute, remix, adapt, build upon this work noncommercially, and license their derivative works on different terms, provided the original work is properly cited and the use is non-commercial. See: http:// creativecommons.org/licenses/by-nc/4.0/ 


\section{REFERENCES}

1. Heurteaux C, Gandin C, Borsotto M, et al. Neuroprotective and neuroproliferative activities of NeuroAid (MLC601, MLC901), a Chinese medicine, in vitro and in vivo. Neuropharmacology 2010;58:987-1001

2. Quintard H, Borsotto M, Veyssiere J, et al. MLC901, a traditional Chinese medicine protects the brain against global ischemia. Neuropharmacology 2011;61:622-31.

3. Moha Ou Maati H, Borsotto M, Chatelain F, et al. Activation of ATP-sensitive potassium channels as an element of the neuroprotective effects of the traditional Chinese medicine MLC901 against oxygen glucose deprivation. Neuropharmacology 2012;63:692-700.

4. Heurteaux $\mathrm{C}$, Widmann $\mathrm{C}$, Moha ou Maati $\mathrm{H}$, et al. NeuroAiD: properties for neuroprotection and neurorepair. Cerebrovasc Dis 2013;35(Suppl 1):1-7.

5. Quintard H, Lorivel T, Gandin C, et al. MLC901, a traditional Chinese medicine induces neuroprotective and neuroregenerative benefits after traumatic brain injury in rats. Neuroscience 2014;277:72-86.

6. Tsai MC, Chang CP, Peng SW, et al. Therapeutic efficacy of NeuroAiD (MLC 601), a traditional Chinese medicine, in experimental traumatic brain injury. J Neuroimmune Pharmacol 2015;10:45-54.

7. Lim YA, Murray LA, Lai MK, et al. NeuroAiD (MLC601) and amyloid precursor protein processing. Cerebrovasc Dis 2013;35(Suppl 1):30-7.

8. Siddiqui FJ, Venketasubramanian N, Chan ES, et al. Efficacy and safety of MLC601 (NeuroAiDR), a traditional Chinese medicine, in poststroke recovery: a systematic review. Cerebrovasc Dis 2013;35 (Suppl 1):8-17.

9. Chen C, Venketasubramanian N, Gan RN, et al. Danqi Piantang Jiaonang (DJ), a traditional Chinese medicine, in poststroke recovery. Stroke 2009;40:859-63.

10. Harandi AA, Abolfazli R, Hatemian A, et al. Safety and efficacy of MLC601 in Iranian patients after stroke: a double-blind, placebo-controlled clinical trial. Stroke Res Treat 2011;2011:721613.

11. Bavarsad Shahripour $\mathrm{R}$, Shamsaei $\mathrm{G}$, Pakdaman $\mathrm{H}$, et al. The effect of NeuroAiD (MLC601) on cerebral blood flow velocity in subjects' post brain infarct in the middle cerebral artery territory. Eur J Intern Med 2011;22:509-13.

12. Kong $\mathrm{KH}$, Wee SK, $\mathrm{Ng} \mathrm{CY}$, et al. A double-blind, placebo-controlled, randomized phase II pilot study to investigate the potential efficacy of the traditional Chinese medicine NeuroAiD (MLC601) in enhancing recovery after stroke (TIERS). Cerebrovasc Dis 2009;28:514-21.

13. Ghandehari K, Izadi Mood Z, Ebrahimzadeh S, et al. NeuroAiD (MLC601) versus piracetam in the recovery of post-infarct homonymous hemianopsia. Neural Regen Res 2011;6:418-22.

14. Venketasubramanian N, Chen CL, Gan RN, et al., CHIMES Investigators. A double-blind, placebo-controlled, randomized, multicentre study to investigate Chinese Medicine NeuroAiD Efficacy on Stroke recovery (CHIMES Study). Int J Stroke 2009;4:54-60.

15. Chen $\mathrm{CL}$, Young SH, Gan HH, et al, CHIMES Study Investigators. Chinese Medicine Neuroaid Efficacy on Stroke Recovery: a double-blind, placebo-controlled, randomized study. Stroke 2013;44:2093-100.

16. Chankrachang S, Navarro JC, de Silva DA, et al., CHIMES Study Investigators. Prognostic factors and treatment effect in the CHIMES Study. J Stroke Cerebrovasc Dis 2015;24:823-7.

17. Navarro JC, Gan HH, Lao AY, et al., CHIMES Study Investigators. Baseline characteristics and treatment response of patients from the Philippines in the CHIMES Study. Int J Stroke 2014;9(Suppl A100):102-5.

18. Chen CL, Venketasubramanian N, Lee CF, et al., CHIMES Study Investigators. Effects of MLC601 on early vascular events in patients after stroke: the CHIMES study. Stroke 2013;44:3580-3.

19. Venketasubramanian N, Young S, Tay SS, et al. Chinese Medicine NeuroAiD Efficacy Stroke Recovery-Extension Study CHIMES-E Study: an observational multicenter study to investigate the longer term efficacy of NeuroAiD in stroke recovery. Cerebrovasc Dis 2013;35(Suppl 1):18-22.

20. Venketasubramanian N, Young SH, Tay SS, et al., CHIMES-E Study Investigators. CHInese Medicine NeuroAiD Efficacy on Stroke
Recovery-Extension Study (CHIMES-E): a Multicenter Study of Long-Term Efficacy. Cerebrovasc Dis 2015;39:309-18.

21. Yeo TT, Chou N. Case report on the use of MLC601 (NeuroAiD) in neurosurgical pathologies [abstract]. Int J Stroke 2010;5(Suppl 2): PO20288.

22. Kumar R, Fadzil F, Soon BH. MLC601 (NeuroAiD) for brain injuries-open label use in a series of patients [abstract]. In: Abstracts of the 14th Asian Australian Congress of Neurological Surgeons; 2015 April 15-18; Jeju Island, Korea: AACNS 2015; Abstract nr 119.

23. Harandi AA, Ashrafi $F$, Tabatabaei M, et al. Efficacy and tolerability of MLC601 in patients with mild to moderate Alzheimer disease who were unable to tolerate or failed benefit from treatment with rivastigmine. Br J Med Med Res 2013;3:341-50.

24. Pakdaman $\mathrm{H}$, Harandi $\mathrm{AA}$, Hatamian $\mathrm{H}$, et al. Effectiveness and safety of MLC601 in the treatment of mild to moderate Alzheimer's disease: a multicenter, randomized controlled trial. Dement Geriatr Cogn Dis Extra 2015;5:96-106.

25. Chen C, Ikram K, Anqi Q, et al., NEURITE Investigators. The NEURoaid II (MLC901) in vascular cognITivE impairment Study (NEURITES). Cerebrovasc Dis 2013;35(Suppl 1):23-9.

26. Navarro JC, Chen CL, Lagamayo PD, et al., CHIMES Investigators. CHIMES-I: subgroup analyses of the effects of NeuroAiD according to baseline brain imaging characteristics among patients randomized in the CHIMES Study. Int J Stroke 2013;8:491-4.

27. https://www.anzctr.org.au/Trial/Registration/TrialReview.aspx? id $=364449$

28. Gan R, Lambert C, Lianting J, et al. Danqi Piantang Jiaonang does not modify hemostasis, hematology and biochemistry in norma subjects and stroke patients. Cerebrovasc Dis 2008;25:450-6.

29. Young SH, Zhao Y, Koh A, et al., CHIMES Investigators. Safety profile of MLC601 (NeuroAiD) in acute ischemic stroke patients: a Singaporean substudy of the Chinese Medicine NeuroAiD Efficacy on Stroke Recovery study. Cerebrovasc Dis 2010;30:1-6.

30. Bavarsad Shahripour R, Hemati A, Hosseinzadeh Maleki A. A randomized trial to assess the long-term safety of NeuroAiD among Caucasian patients with acute ischemic stroke. Chin J Integr Med 2014:20:812-17.

31. Brott T, Adams HP Jr, Olinger CP, et al. Measurements of acute cerebral infarction: a clinical examination scale. Stroke 1989;20: 864-70.

32. Teasdale G, Jennett B. Assessment of coma and impaired consciousness: a practical scale. Lancet 1974;2:81-4.

33. Rankin J. Cerebral vascular accidents in patients over the age of 60 . II. Prognosis. Scot Med J 1957;2:200-15.

34. Katzman R, Brown T, Fuld P, et al. Validation of a short Orientation-Memory-Concentration Test of cognitive impairment. Am J Psychiatry 1983;140:734-9.

35. http://www.who.int/medicines/areas/quality_safety/safety_efficacy/ WHOcausality_assessment.pdf

36. Hanley JA, Lippman-Hand A. If nothing goes wrong, is everything alright? JAMA 1983;249:1743-5.

37. Eyspach E, Lefering R, Kum CK, et al. Probability of adverse events that have not yet occurred: a statistical reminder. $B M J$ 1995;311:619-20.

38. Evans SJ, Waller PC, Davis S. Use of proportional reporting ratios (PRRs) for signal generation from spontaneous adverse drug reaction reports. Pharmaco epidemiol Drug Saf 2001;10:483-6.

39. Bate A, Lindquist M, Edwards IR, et al. A Bayesian neural network method for adverse drug reaction signal generation. Eur J Clin Pharmacol 1998;54:315-21.

40. Bate A, Lindquist M, Edwards IR, et al. A data mining approach for signal detection and analysis. Drug Saf 2002;25:393-7.

41. Bate A. Bayesian confidence propagation neural network. Drug Saf 2007;30:623-5.

42. Hazell L, Shakir SA. Under-reporting of adverse drug reactions: a systematic review. Drug Saf 2006;29:385-96.

43. Olsson S, Pal SN, Stergachis A, et al. Pharmacovigilance activities in 55 low- and middle-income countries: a questionnaire-based analysis. Drug Saf 2010;33:689-703.

44. Gliklich RE, Dreyer NA. Registries for Evaluating Patient Outcomes: a user's guide. 2nd edn. Rockville, MD: Agency for Healthcare Research and Quality, 2010:79-101. 